\title{
Solidarität von allen Seiten beleuchtet
}

Anna Sax

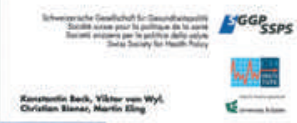

Brennpunkt Solidarität

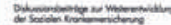

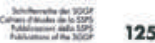

Konstantin Beck,

Viktor von Wyl,

Christian Biener,

Martin Eling

Brennpunkt Solidarität

Diskussionsbeiträge zur Weiterentwicklung der Sozialen Krankenversicherung Bern: Schriftenreihe der SGGP Band 125; 2014.

180 Seiten. 40 CHF für

Mitglieder der SGGP,

52 CHF für Nicht-Mitglieder

ISBN 978-3-85707-125-6
Die Solidarität in der sozialen Krankenversicherung ist seit der Einführung des geltenden KVG vor bald 20 Jahren ein politischer Dauerbrenner. Doch wie manifestiert sich Solidarität? Wer ist mit wem solidarisch (bzw. sollte es sein): die Jungen mit den Alten? Die Gesunden mit den Kranken? Die Reichen mit den Armen? Die Thurgauerinnen mit den Genfern? Was geschieht, wenn man Solidarität nicht nur als Momentaufnahme, sondern dynamisch über die Zeit hinweg betrachtet? Und was unterscheidet interkantonale von intergenerationellen Quersubventionen?

Der neue Band aus der Schriftenreihe der SGGP bringt interessante und teilweise neue ökonomische Erkenntnisse zu diesem ebenso wichtigen wie schwer fassbaren Thema. Vier Ökonomen präsentieren bekannte und weniger bekannte Fakten, analysieren politische Reformen, stellen neue $\mathrm{Zu}$ sammenhänge her und schlagen Lösungen vor. Finanziert wurde der Sammelband von der Universität St. Gallen und vom CSS Institut für Gesundheitsökonomie.

In Kapitel 2 analysieren die St. Galler Versicherungs-

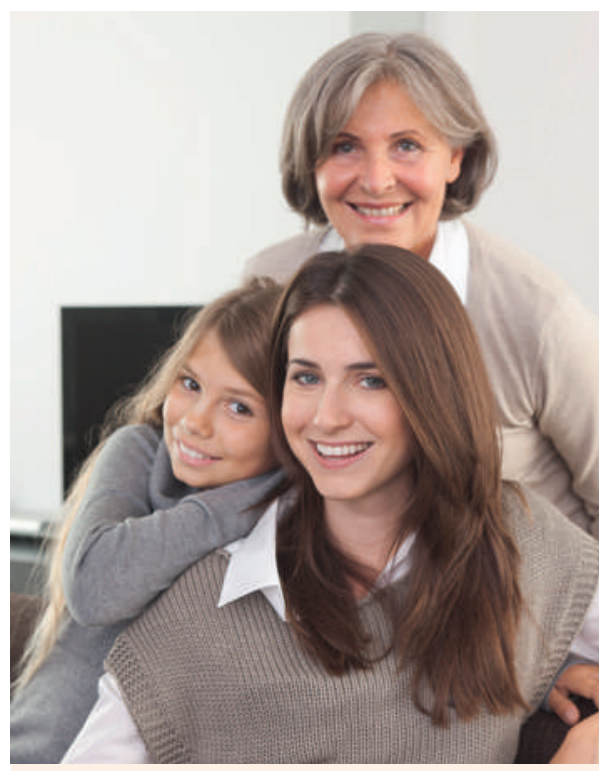

Wichtiges Thema des Buchs: Generationenvertrag und Solidarität zwischen Alt und Jung in der Krankenversicherung. daten von 840000 Versicherten - aus welchen Datenbeständen bleibt unklar - untersuchen, an wen die Prämienrechnungen adressiert sind, d.h. wer sie letztlich bezahlt: Es zeigt sich, dass die Altersgruppen zwischen 36 und 65 Jahren «in beträchtlichem Ausmass für andere Versicherte aufkommen», hauptsächlich wohl für eigene Kinder, Jugendliche oder Ehepartner. Überraschend kommt heraus, dass die effektive Prämienlast der verschiedenen Altersgruppen weitgehend mit den altersspezifischen mittleren Einkommen korrespondiert.

Die CSS-Ökonomen schlagen vor allem im Bereich des Risikoausgleichs neue Reformen vor. So würde zum Beispiel eine Senkung der Risikoabgabe für junge Erwachsene die Effizienz der Finanzierung der Grundversicherung verbessern, wie Beck in Kapitel 5 aufzeigt. Zudem könnte mit einem neuen mehrstufigen Verfahren verhindert werden, dass über die Hälfte der Einsparungen, die Versicherte in Hausarztmodellen erzielen, über den Risikoausgleich an «normale» Versicherte umverteilt werden. ökonomen Biener und Eling retrospektiv die Umverteilung zwischen Jung und Alt, wobei die Formeln und Grafiken zum Teil schwer zu interpretieren sind und die meisten Lesenden sich auf die Schlussfolgerungen werden verlassen müssen: Die zunehmende Umverteilung von Jung nach Alt war in der Vergangenheit vor allem auf den Mengen-Effekt zurückzuführen, während die Demographie und die Preise eine eher untergeordnete Rolle spielten. Der zunehmende Anteil alter Menschen schlägt also weniger stark als erwartet auf die Prämien. Auch die mathematischen Exkurse in Kapitel 3 eignen sich eher für «Angefressene». Von Wyls Beitrag ist aber auch für Laien durchaus lesenswert, denn er schlägt eine dynamische Interpretation der Solidarität vor: Die Jungen von heute haben über den Risikoausgleich zwar einen hohen Solidaritätsbeitrag zu leisten, doch auch sie werden älter. Über den ganzen Lebenszyklus hinweg betrachtet könnte ihre Bilanz sogar positiv ausfallen.

Einen originellen Ansatz wählen von Wyl und Beck in Kapitel 4, wo sie aufgrund der Abrechnungs-
Überhaupt ist der Risikoausgleich gemäss seinem langjährigen Fürsprecher und Entwickler Beck etwas hochtrabend als «die Mutter aller Reformen» betitelt, Hauptgegenstand der meisten Beiträge. Und es zeigt sich in der Tat, dass hier auch nach der neuesten beschlossenen Gesetzesreform noch weitere Verbesserungen möglich sind.

Gegen Ende des Bandes rückt die Sicht der Versicherer etwas stärker ins Zentrum, etwa wenn es darum geht, die positiven Auswirkungen der Weiterentwicklung des Risikoausgleichs darzustellen. Von Wyl hält es für erwiesen, dass die Versicherer in der gewünschten Weise auf die neuen Anreize reagieren, die mit den Revisionen gesetzt worden sind. Den Schlusspunkt setzt Beck mit einem fulminanten und sehr lesenswerten Beitrag zum Thema «falsche Prämienberechnungen» der Versicherer und Interessenlagen der kantonalen Regierungen. Und er schafft es tatsächlich, eine Lösung zu präsentieren, die sowohl den Versicherern das Geschäft wie auch den Regierungsräten die Wiederwahl erleichtert. 